\title{
Simulation of Logging-while-drilling Tool Response Using Integral Equation Fast Fourier Transform
}

\author{
Xiang-Yang SUN ${ }^{1, a}$, Bao-Xin $\mathrm{LI}^{1}$, Yong-Peng ZHAO ${ }^{1}$ \\ ${ }^{1}$ High tech Zone(West), Chengdu, Sichuan, China Xi yuan Avenue, No. 2006, University of Electronic Science and \\ Technology
}

\begin{abstract}
We rely on the volume integral equation (VIE) method for the simulation of loggingwhile-drilling (LWG) tool response using the integral equation fast Fourier transform (IE-FFT) algorithm to accelerate the computation of the matrix-vector product in the iterative solver. Depending on the virtue of the Toeplitz structure of the interpolation of the Green's function on the uniform Cartesian grids, this method uses FFT to calculate the matrix-vector multiplication. At the same time, this method reduce the memory requirement and CPU time. In this paper, IEFFT method is first used in the simulation of LWG. Numerical results are presented to demonstrate the accuracy and efficiency of this method. Compared with the Moment of Method (MOM) and other fast algorithms, IE-FFT have distinct advantages in the fact of memory requirement and CPU time. In addition, this paper study the truncation, mesh elements, the size of the interpolation grids of IE-FFT and dip formation, and give some conclusion with wide applicability.
\end{abstract}

\section{Introduction}

Logging-while-drilling tools [1-2] are based on the theory of waves and fields in inhomogenous media. In the field of petroleum well drilling and logging, electromagnetic wave resistivity sensors are frequently used to provide an indication of the electrical resistivity of rock formations surrounding an earth borehole. Such information regarding resistivity is useful in ascertaining the presence or absence of oil. A typical electromagnetic wave resistivity tool comprises a transmitter antenna and a pair of receiver antennas located at different distance from the transmitter antenna along the axis of the tool. The transmitter antenna is used to transmit electromagnetic waves. Due to waves received by two receiver antennas propagate through different distance, they are absorbed by the surrounding earth formation with different degree. So the voltages in the two receiver antennas have different phases and amplitudes. This is the reason that we use the phase different (PD) and amplitude ratio (AR) of voltages on the two receiver antennas to analyze the rock formation.

Simulation of LWD Tool response is of great importance for optimization and design the new tools, and interpretation the earth formation and son on. Numerical methods such as finite element method (FEM)[3-5]' finite difference time domain (FDTD), finite difference method (FDM) and moment of method (MOM) are flexible in dealing with 3-D formation. By contrast, the volume integral equation only require to divide the areas that are different with the background, so it has less

\footnotetext{
${ }^{\text {a }}$ Corresponding author: sunxiangyang@uestc.edu.cn
} 
unknowns than FEM. One conventional method of solution for the volume integral equation is the method of moment (MOM). However, MOM methods are limited to relatively small problems because of their excessive CPU time and memory requirements. Therefore, Zhong Qing Zhang successfully proposed to improve the efficiency of induction logging problems with the CG-FFT method[6-10]. While CG-FFT require the uniform elements, it will create too many basis functions. Another method is MOM combine with multi-level adaptive cross approximation (MOM+MLACA)[11-14] used by the group of Zaiping Nie in UESTC, while MLACA is one method of pure mathematics. And the capacity to reduce computing time and memory is limited. In this paper, IE-FFT [15-16] is first used to solve the problem of the simulation of LWD tool response. IE-FFT method depends on the interpolation of the Green's function on the uniform Cartesian grids. And it takes advantages of the Fast Fourier Transform (FFT) to calculate the matrix-vector multiplication without needs to insure the uniform mesh elements. The superiority of IE-FFT is more attractively with the increase of the unknowns. This method is proved to be effective by contrast with the NMM, MOM, MOM+MLACA and MOM combine with multi-level adaptive cross approximation and singular value decomposition (MOM+MLACA+SVD). And it is clear that IE-FFT has significant advantages for reducing the memory requirement and CPU time.

\section{The volume integral equation}

In the model of the simulation of logging response, most of the area is homogeneous medium regarding as the background. The background is characterized by distribution of permittivity $\varepsilon_{b}$ and conductivity $\delta_{b}$, and the permeability is assumed constant $\mu_{b}=\mu_{\mathrm{o}}$. While the inhomogenous formation are characterized by distribution of permittivity $\varepsilon(r)$ and conductivity $\delta(r)$, and the permeability is assumed constant $\mu_{b}=\mu_{\mathrm{o}}$. Assumed that $\mathrm{E}^{i}$ is the excitation field, and the equivalent volume current is $J_{v}$ in inhomogenous formation. The scattered field $E_{V}^{\text {sca }}$ is the contribution of the volume current $J_{v}$, which can be calculated by

$$
E_{V}^{\mathrm{sca}}(r)=i w A_{v}(r)-\nabla \varphi_{v}(r)
$$

where

$$
\begin{aligned}
& A_{\mathrm{v}}(r)=\frac{\mu_{b}}{4 \pi} \int_{v} J_{v}(r) \frac{e^{i k_{b}\left|r-r^{\prime}\right|}}{\left|r-r^{\prime}\right|} d V^{\prime} \\
& \varphi_{\mathrm{v}}(r)=\frac{1}{4 \pi i \omega \varepsilon_{b}} \int_{V} \nabla \cdot J_{V}(r) \frac{e^{i k_{b}\left|r^{\prime}\right|}}{\left|r-r^{\prime}\right|} d V^{\prime}
\end{aligned}
$$

where $\varepsilon_{\mathrm{b}}^{\prime}$ is the complex permittivity of background and $\mathrm{k}_{b}$ is the wave-number in the background medium given by

$$
\begin{aligned}
& \varepsilon_{\mathrm{b}}^{\prime}=\varepsilon_{b}+i \delta_{b} / \omega \\
& \mathrm{k}_{b}=\omega \sqrt{\mu_{b}\left(\varepsilon_{b}+i \delta_{b} / \omega\right)}
\end{aligned}
$$

In the volume region 


$$
D(\mathrm{r}) / \varepsilon(r)=E_{V}^{s c a}(r)+E^{i n c}(r) \quad r \in V
$$

The unknown electric flux density can be expanded as

$$
i w D(\mathrm{r})=\sum_{n=1}^{N v} f_{n}^{v}(r)
$$

while

$$
I_{v}(\mathrm{r})=i w\left(k_{b}^{2} / k^{2}(r)-1\right) D(r) \quad \sum_{n=1}^{N \underline{N}} I_{n}^{N v} \chi(r) f_{n}^{v}(r)
$$

where $\chi$ is the contrast of the inhomogenous formation

$$
\chi(r)=k_{b}^{2} / k^{2}(r)-1
$$

Substituting (7) (8) into (6) and using the GalerKin's technique yield a linear system with $\mathrm{N}$ unknowns

$$
\sum_{n=1}^{N v} I_{n}^{V}\left[f_{n}^{V} / i w \varepsilon f_{m}^{V}-i w\left(A_{n}^{V} f_{m}^{V}\right)+\left(\nabla \phi_{n}^{V} f_{m}^{V}\right)\right]=\left(E^{i} f_{m}^{V}\right) \quad \mathrm{m}=1,2 \ldots \ldots \mathrm{N}_{V}
$$

Where

$$
\begin{gathered}
\phi_{n}^{V}(r)=\frac{1}{4 \pi i w \varepsilon_{b}} \int_{\Sigma T_{n}^{ \pm}} \nabla \cdot \chi f_{n}^{V} \frac{e^{i k_{b}\left|r^{\prime}\right|}}{\left|r-r^{\prime}\right|} d r \\
=\frac{1}{4 \pi i w \varepsilon_{b}} \int_{\Sigma T_{n}^{ \pm}} \chi \nabla \cdot f_{n}^{V} \frac{e^{i k_{b}\left|r-r^{\prime}\right|}}{\left|r-r^{\prime}\right|} d r-\frac{\chi_{n}^{+}-\chi_{n}^{-}}{4 \pi i w \varepsilon_{b}} \int_{a_{n}} \frac{e^{i k_{b}\left|r-r^{\prime}\right|}}{\left|r-r^{\prime}\right|} d s \\
\nabla \phi_{n}^{V} f_{m}^{V}=\int_{s} \phi f_{m}^{V}(r) \cdot n d S-\int_{V} \phi \nabla \cdot f_{m}^{V}(r) d V \\
=\int_{V} \phi \nabla \cdot f_{m}^{V}(r) d V \quad \mathrm{~T}_{n}^{ \pm} \in V \\
\int_{S} \phi d S-\int_{V} \phi \nabla \cdot f_{m}^{V}(r) d V \quad \mathrm{~T}_{n}^{ \pm} \in V
\end{gathered}
$$

Let

$$
Z_{1 m n}=-i w\left(A_{n}^{V} f_{m}^{V}\right)+\left(\nabla \phi_{n}^{V} f_{m}^{V}\right)
$$

\section{IE-FFT method in the simulation of Iwd tool response}

The IE-FFT method is irregular mesh-based grid algorithm. The uniform grids are used for interpolating the Green's function. The action of Green's function on a vector can be computed efficiently using the FFT. For near interactions, correction of impedance is must, since the accuracy of the sampled representation is insufficient. The Green's function interpolated by Lagrangian polynomials can be approximated as 


$$
G\left(r, r^{\prime}\right) \cong \beta(r) \cdot G \cdot(\beta(r))^{T}
$$

where

$$
\begin{aligned}
& \beta(r)=\left[\begin{array}{ll}
\beta_{0}^{P}(r) \beta_{1}^{P}(r) \ldots \beta_{N_{g}-1}^{P}(r)
\end{array}\right] \\
& =\left[\begin{array}{c}
\beta_{0}^{P}(x) \beta_{0}^{P}(y) \beta_{0}^{P}(z) \\
\beta_{1}^{P}(x) \beta_{1}^{P}(y) \beta_{1}^{P}(z) \\
\beta_{N_{X}-1}^{P}(x) \beta_{N_{y}-1}^{P}(y) \beta_{N_{z}-1}^{P}(z)
\end{array}\right] \\
& G=\left[\begin{array}{cccc}
G_{0,0} & G_{0,1} & \ldots & G_{0, N_{g}-1} \\
G_{1,0} & G_{1,1} & \ldots & G_{1, N_{g}-1} \\
\ldots & \ldots & \ldots & \ldots \\
G_{N_{g}-1,0} & G_{N_{g}-1,1} & \ldots & G_{N_{g}-1, N_{g}-1}
\end{array}\right]
\end{aligned}
$$

where $N_{x}, N_{y}, N_{z}$ are the number of grid points in $x, y$ and $z$ coordinates. $N_{g}$ is the total number of the grid points as $N_{g}$. Substituting (14) into (13)

$$
Z_{m n}=-i w \mu_{b}\left\{\begin{array}{l}
\Pi_{3} \cdot G \cdot\left(\Pi_{1}\right)^{T}-\frac{1}{k_{b}^{2}} \Pi_{4} \cdot G \cdot\left(\Pi_{2}\right)^{T}+\frac{1}{k_{b}^{2}} \Pi_{4} \cdot G \cdot\left(\Pi_{5}\right)^{T} \\
+\frac{1}{k_{b}^{2}} \Pi_{6} \cdot G \cdot\left(\Pi_{2}\right)^{T}-\frac{1}{k_{b}^{2}} \Pi_{6} \cdot G \cdot\left(\Pi_{5}\right)^{T}
\end{array}\right\}
$$

where

$$
\begin{gathered}
\Pi_{1}=\int_{V_{n}} \chi(r) \cdot f_{n}^{V}(r) \cdot \beta_{l}^{p}(r) d r \\
\Pi_{2}=\int_{V_{n}} \chi(r) \nabla \cdot f_{n}^{V}(r) \cdot \beta_{l}^{p}(r) d r \\
\Pi_{3}=\int_{V_{n}} f_{n}^{V}(r) \cdot \beta_{l}^{p}(r) d r \\
\Pi_{4}=\int_{V_{n}}^{\nabla} \cdot f_{n}^{V}(r) \cdot \beta_{l}^{p}(r) d r \\
\Pi_{5}=\left(\chi_{n}^{+}-\chi_{n}^{-}\right) \int_{V_{n}} f_{n}^{V}(r) \cdot \beta_{l}^{p}(r) d r \\
\Pi_{3}=\int_{S_{n}} \beta_{l}^{p}(r) d r
\end{gathered}
$$


When the near interactions are computed via the IE-FFT method, the results are highly erroneous. The correction value can be computed as the difference between the value directly computed by the MOM and the value computed via the IE-FFT algorithm

$$
Z=Z_{M O M}^{\text {near }}-Z_{I E-F F T}^{\text {near }}+Z_{I E-F F T}=Z^{\text {corr }}+Z_{I E-F F T}
$$

the matrix vector product can be compute via the FFT

$$
\begin{aligned}
& V= \\
& Z^{\text {corr }} \cdot I-i w \mu_{b}\left(\Pi_{3} \cdot \operatorname{IFFT}\{F F T(G)\} \cdot F F T\left[\left(\Pi_{1}\right)^{T} \cdot I\right]-\frac{1}{k_{b}^{2}} \Pi_{4} \operatorname{IFFT}(F F T(G) \cdot\right. \\
& \left.\operatorname{FFT}\left[\left(\Pi_{2}\right)^{T} \cdot I\right]\right)+\frac{1}{k_{b}^{2}} \Pi_{4} \operatorname{IFFT}\left(F F T(G) \cdot \operatorname{FFT}\left[\left(\Pi_{5}\right)^{T} \cdot I\right]\right)+\frac{1}{k_{b}^{2}} \Pi_{6} \\
& \left.\operatorname{IFFT}\left(\operatorname{FFT}(G) \cdot \operatorname{FFT}\left[\left(\Pi_{2}\right)^{T} \cdot I\right]\right)-\frac{1}{k_{b}^{2}} \Pi_{6} \operatorname{IFFT}\left(F F T(G) \cdot \operatorname{FFT}\left[\left(\Pi_{5}\right)^{T} \cdot I\right]\right)\right)
\end{aligned}
$$

\section{Numerical results}

eg1.The tolerance of 0.001 use GMRES iterations. The Green's function interpolate by the 2nd order Lagrangian polynomials. The size of interpolation grids is $0.1 \lambda$ and the near gate for correction is $0.1 \sqrt{2} \lambda$ ( $\lambda$ is the wavelength of the background medium ). The inhomogenous formation has a thickness of $3 \mathrm{~m}$ and the conductivity of $0.1 \mathrm{~S} / \mathrm{m}$. The borehole has a $0.127 \mathrm{~m}$ radius and filled with mud with conductivity of $3.0 \mathrm{~S} / \mathrm{m}$. The mud conductivity is treated as background medium. The working frequency of the LWD tool is $500 \mathrm{KHz}$. The two receiver antennas are located at $1.0 \mathrm{~m}$ and $1.5 \mathrm{~m}$ away from the transmitter antenna. The total unknowns is 47854 . The number of spatial samples are 41 . The result is obtained on the computer with $3.3 \mathrm{GHz}, 8$ core.

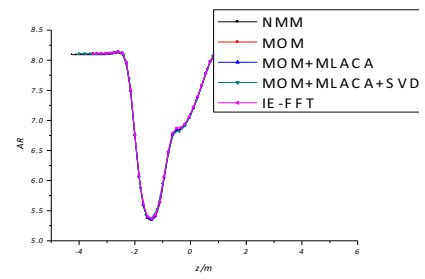

Figure 1 (a)Amplitude Ratio

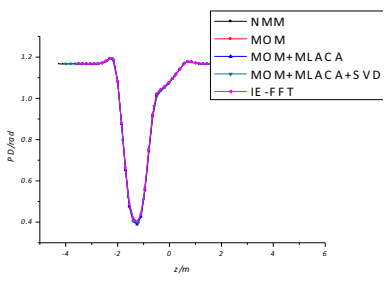

Figure 1 (b) Phase Difference

Table1 The contrast of the CPU time and memory requirement of MOM, MOM+MLACA

\begin{tabular}{|l|l|l|}
\hline & $\begin{array}{l}\text { Memory } \\
(\mathrm{G})\end{array}$ & $\begin{array}{l}\text { Time } \\
(\mathrm{S})\end{array}$ \\
\hline MOM & 34 & 38795 \\
\hline MOM+MLACA & 9.3 & 994 \\
\hline MOM+MLACA+SVD & 2.7 & 444 \\
\hline IE-FFT & 2.6 & 274 \\
\hline
\end{tabular}

eg2.The tolerance of 0.001 use GMRES iterations. The Green's function interpolate by the 2nd order Lagrangian polynomials. The size of interpolation grids is $0.1 \lambda$ and the near gate for correction 
is $0.1 \sqrt{2} \lambda$ ( $\lambda$ is the wave length of the background medium). The inhomogenous formation has a thickness of $6 \mathrm{~m}$ and the conductivity of $0.3 \mathrm{~S} / \mathrm{m}$. The borehole has a $0.1 \mathrm{~m}$ radius and filled with mud with conductivity of $8.0 \mathrm{~S} / \mathrm{m}$. The mud conductivity is treated as background medium. The working frequency of the LWD tool is $450 \mathrm{KHz}$. The two receiver antennas are located at $1.0 \mathrm{~m}$ and $1.5 \mathrm{~m}$ away from the transmitter antenna. The total unknowns is 414926 . The number of spatial samples are 41 . The result is obtained on the computer with $2.27 \mathrm{GHz}, 16$ core.

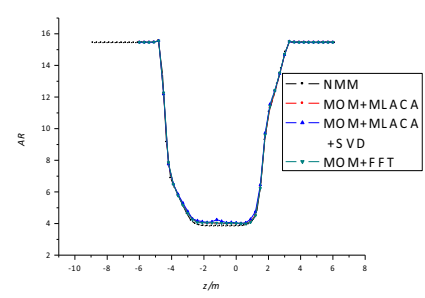

Figure 2 (a)Amplitude Ratio

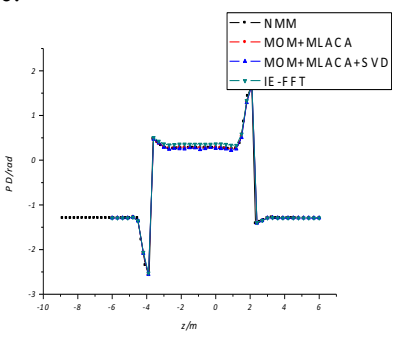

Figure 2 (b) Phase Difference

Table2 The contrast of the CPU time and memory requirement of MOM, MOM+MLACA, $\mathrm{MOM}+\mathrm{MLACA}+\mathrm{SVD}$ and IE-FFT in Fig2

\begin{tabular}{|l|l|l|}
\hline & Memory $(\mathrm{G})$ & Time $(\mathrm{S})$ \\
\hline MOM+MLACA & 207 & $30945(8.6 \mathrm{~h})$ \\
\hline MOM+MLACA+SVD & 43 & $8476(2.4 \mathrm{~h})$ \\
\hline IE-FFT & 16 & $6781(1.9 \mathrm{~h})$ \\
\hline
\end{tabular}

The numerical results proved the effective of the simulation of LWD tool of response with IE-FFT. By contrast with the MOM, MOM+MLACA and MOM+MLACA+SVD, it clear that IE-FFT method has its own superiority in the fact of the memory requirement and CPU time. And the advantages will be more attractive with the increase of the unknowns.

\section{Conclusion}

The IE-FFT methods is irregular mesh-based grid algorithm. The IE-FFT methods is the interpolation of the Green's function on the uniform Cartesian tensor grids by Lagrangian polynomials. With the virtue of the Toeplitz structure of the Green's function, this method uses FFT to accelerate the matrixvector product. The advantages of FFT are that it makes the computational complexity of $\mathrm{O}\left(\mathrm{N}^{2}\right)$ tend to $\mathrm{O}(\mathrm{N} \log \mathrm{N})$ and it makes the memory requirement of $\mathrm{O}\left(\mathrm{N}^{2}\right)$ tend to $\mathrm{O}(\mathrm{N})$. So IE-FFT can heavily reduce the memory requirement and CPU time. Compared with MOM, MOM+MLACA and $\mathrm{MOM}+\mathrm{MLACA}+\mathrm{SVD}$, it is proved that IE-FFT method has advantages in CPU time and memory requirement using solving the problem of logging. And compared with NMM, this method can use to solve the problem of $3-\mathrm{D}$.

\section{References}

1. H. G. Doll. Introduction to induction logging and application to logging of wells drilled with oilbased mud[J].Journal of Petroleum Technology, 1949, 1(6):148-162

2. Weng Cho Chew, Zaiping Nie, Qing-Huo Liu, Barbara Anderson. An efficient solution for the response of electrical well logging tool in a complex environment[J].IEEE Transaction on Geoscience and Remote Sensing, 1991, 29(2):309-313

3. B. Anderson, S. K. Chang. Synthetic induction logs by the finite element method[J].Log Anal., 1982, 23:17-29[J]. SPWLA 23rd Annual Logging Symposium, 1982, 23(1):17-29 
4. S. K. Chang, B. Anderson. Simulation of induction logging by the finite element method[J].Geophysics, 1984, 49(11): 1943-1958

5. Sun Xiang-Yang, Nie Zai-Ping. 3-D Modeling of Electromagnetic Induction in Anisotropy Media Using Finite Element Method[J]. Antennas and Propagation Society International Symposium, 2008, AP-S 2008(1):1-4

6. Zhong Qing Zhang, Qing Huo Liu . The Hybrid Extended Born Approximation and CG-FFHT method for axisymmetric media[J].Antennas and Propagation Society International Symposium, 2001, 39(4): 710- 717

7. Qing Huo Liu ,Zhong Qing Zhang, Xue Min Xu . The Hybrid Extended Born Approximation and CG-FFHT method for electromagnetic induction problems[J].IEEE Transactions on Geo-science and Remote Sensing, 2001, 39(2):347-355

8. Zhong Qing Zhang, Qing Huo Liu. The Hybrid Extended Born Approximation and CG-FFHT method for axisymmetric media[J].IEEE Transactions on Geo-science and Remote Sensing, 2001, 39(4):710-717

9. Zhong Qing Zhang, Qing Huo Liu. Simulation of 3D EM fields by a weak-form biconjugate gradient FFT method [J].Antennas and Propagation Society International Symposium, 2001, 4(1):626-629

10. C.Garcia, Y. Alvarez. The adaptive cross approximation algorithm applied to a volumetric method-of-moments for electromagnetic analysis of inhomogeneous bodies[J].Antennas and Propagation Society International Symposium, 2010, 2010 IEEE(1):1-4

11. M.Astner, H. D. Bruns.Application of a hierarchical SVD-ACA compression technique to nearfield calculation of monopole antennas[J].EMC International Symposium, 2007, EMC 2007(1):16

12. Kezhong Zhao, Marinos N. Vouvakis. The adaptive cross approximation algorithm for accelerated method of moments computations of EMC Problems[J].IEEE Trans.EMC, 2005, 47(4):763-773

13. Peng Li. Numerical simulation of response of electrical logging based on integral equation method[Master's thesis]. Chengdu: School of Electronic Engineering of University of Electronic Science and Technology of China, 2012

14. Seung Mo Seo, Jin-Fa Lee.A fast IE-FFT algorithm for solving PEC scattering problems[J].IEEE Transaction on Magnetic, 2008, 44(6):1398-1401

15. Nilufer A.Ozdemir,Jin-Fa LeeIE-FFT algorithm for a non-conformal volume integral equation for electromagnetic scattering from dielectric objects[J].IEEE Transaction on Magnetic, 2008, 44(6):1398-1401 